Article

\title{
Risk Prediction of Second Primary Endometrial Cancer in Obese Women: A Hospital-based Cancer Registry Study
}

\author{
Chi-Chang Chang ${ }^{1,2}$, Chun-Chia Chen ${ }^{3,4, *}$, Cheewakriangkrai ${ }^{5, *}$, Ying-Chen Chen ${ }^{1}$ and Shun-Fa Yang ${ }^{3}$ \\ 1 School of Medical Informatics, Chung Shan Medical University \& IT office, Chung Shan Medical University \\ Hospital, Taichung 40201, Taiwan. \\ 2 Department of Information Management, Ming Chuan University, Taoyuan 33300, Taiwan. \\ 3 Institute of Medicine, Chung Shan Medical University, Taichung 40201, Taiwan. \\ 4 Division of Plastic Surgery, Department of Surgery, Chung Shan Medical University Hospital, Taichung \\ 40201, Taiwan. \\ 5 Division of Gynecologic Oncology, Department of Obstetrics and Gynecology, Faculty of Medicine, Chiang \\ Mai University, Chiang Mai, Thailand. \\ * Correspondence: chenjica@gmail.com (Chun-Chia Chen), Tel: +886-4-24730022; chalong.c@cmu.ac.th \\ (Chalong Cheewakriangkrai), Tel: +66 53935552.
}

\begin{abstract}
Due to the high effectiveness of cancer screening and therapies, the diagnosis of second primary cancers (SPCs) has increased in women with endometrial cancer (EC). However, there's no previous literature mentioned about adequate evidence to support screening for SPCs in endometrial cancer. This study was aimed to develop effective risk prediction models of second primary endometrial cancer in women with obesity (Body-mass index; BMI > 25) and this study includes datasets of the incidence of SPCs and the other risks of SPCs in 4480 primary cancer survivors by a hospital-based cancer registry database. In our study, we found the obesity played a key role in SPCs. There're 10 independent variables used as predicting variables, which corelated to obesity should be monitored for the early detection of SPCs in endometrial cancer. In conclusion, it is a promising SPCs prediction. The proposed scheme can support the important influence of obesity and clinical data representations in all cases after primary treatments. Our results suggested that obesity is still a crucial risk factor to SPCs in endometrial cancer.
\end{abstract}

Keywords: second primary cancers (SPCs); endometrial cancer (EC); risk prediction.

\section{Introduction}

Endometrial cancer (EC) is the most common gynecological malignancy, and its incidence is rising alongside the growing prevalence of obesity.[1]

Endometrial cancer effected women worldwide, and resulted in an estimation of 42 000 deaths from this cancer.[2] The most endometrial cancer happens after the menopause, with a relation of long-term exposure to unopposed estrogens. For average, the overall 5 -year survival is around $80 \%$. Overweight (defined as body-mass index [BMI] of at least $25 \mathrm{~kg} / \mathrm{m} 2$ ) also plays an important risk factor present in $50 \%$ of endometrial cancers. A BMI above $25 \mathrm{~kg} / \mathrm{m} 2$ doubles a woman's risk of endometrial cancer, and a BMI above $30 \mathrm{~kg} / \mathrm{m} 2$ triples the risk.[3,4] Therefore, understanding the key mechanisms driving endometrial carcinogenesis in primary endometrial cancer may affect second primary endometrial cancer diagnoses if aimed at those at greatest risk. An understanding of the correlation between obesity and SPCs is critical in developing such prevention strategies.[1] 
In Taiwan Cancer Registry database, there're 9 variables recorded as clinical prognostic factors, (1) Age at Diagnosis, (2) Grade/differentiation, (3) Tumor size, (4) Clinical stage Group, (5) Pathologic Stage Group, (6) Surgical Margins involvements of The Primary Site, (7) Date of first surgical procedure, (8) Sequence of Radiotherapy and Surgery, (9) Sequence of Locoregional Therapy and Systemic Therapy. In this study, we proposed those factors and BMI could be the important predictors in SPCs of endometrial cancers. Therefore, the purpose of the analysis is to identify the most important risk factors from 10 predictors: (Table 1\&2).

Table 1. The important variables associated with endometrial cancer.

\begin{tabular}{ll}
\hline Rank & Variable Name \\
\hline 1 & Clinical stage Group \\
2 & Tumor size \\
2 & Pathologic Stage Group \\
2 & Date of first surgical procedure \\
5 & BMI \\
6 & Age at Diagnosis \\
7 & Sequence of Locoregional Therapy and Systemic Therapy \\
8 & Grade/differentiation \\
8 & Surgical Margins of The Primary Site \\
10 & Sequence of RT and Surgery \\
\hline
\end{tabular}

We suggest potential prevention strategies and make a case for the need for risk prediction models that identify specific groups of women at a particularly high risk of endometrial cancer for whom risk-reducing interventions are likely to have a significant impact. 
Table 2. Subject Demographics of all primary endometrial cancers.

\begin{tabular}{|c|c|c|c|}
\hline \multirow[t]{2}{*}{ Characteristics } & \multicolumn{3}{|c|}{ Endometrial cancer $(\mathrm{N}=1560)$} \\
\hline & Without SPCs & With SPCs & p value \\
\hline $\mathrm{N}(\%)$ & $1040(66.7 \%)$ & $520(33.3 \%)$ & \\
\hline Age at Diagnosis & & & $<0.001^{* *}$ \\
\hline$<50$ year-old & $372(35.7 \%)$ & $140(26.9 \%)$ & \\
\hline$\geq 50$ year-old & $668(64.3 \%)$ & $380(73.1 \%)$ & \\
\hline Grade/Differentiation & & & $0.014^{*}$ \\
\hline 1,2 & $705(67.8 \%)$ & $320(61.5 \%)$ & \\
\hline others & $335(32.2 \%)$ & $200(38.5 \%)$ & \\
\hline Tumor Size & & & $<0.001^{* *}$ \\
\hline$<2 \mathrm{~cm}$ & $262(25.2 \%)$ & $220(42.3 \%)$ & \\
\hline$\geq 2 \mathrm{~cm}$ & $778(74.8 \%)$ & $300(57.7 \%)$ & \\
\hline Clinical Stage & & & $<0.001^{* *}$ \\
\hline$<\mathrm{II}$ & $838(80.6 \%)$ & $280(53.8 \%)$ & \\
\hline$\geq \mathrm{II}$ & $202(19.4 \%)$ & $240(46.2 \%)$ & \\
\hline Pathologic Stage & & & $<0.001^{* *}$ \\
\hline$<\mathrm{II}$ & $834(80.2 \%)$ & $480(92.3 \%)$ & \\
\hline$\geq \mathrm{II}$ & $206(19.8 \%)$ & $40(7.7 \%)$ & \\
\hline Surgical Margins involvement & & & 0.405 \\
\hline No & $947(91.1 \%)$ & $480(92.3 \%)$ & \\
\hline Yes & $93(8.9 \%)$ & $40(7.7 \%)$ & \\
\hline Surgical procedure & & & $<0.001^{* *}$ \\
\hline No & $40(3.8 \%)$ & $0(0.0 \%)$ & \\
\hline Yes & $1000(96.2 \%)$ & $520(100.0 \%)$ & \\
\hline Sequence of Radiotherapy/Surgery & & & 0.689 \\
\hline No & $611(58.8 \%)$ & $300(42.9 \%)$ & \\
\hline Yes & $429(41.2 \%)$ & $220(57.1 \%)$ & \\
\hline Sequence of Locoregional/Systemic therapy & & & $<0.001^{* *}$ \\
\hline No & $740(71.2 \%)$ & $320(57.7 \%)$ & \\
\hline Yes & $300(28.8 \%)$ & $200(42.3 \%)$ & \\
\hline BMI & & & $0.001^{*}$ \\
\hline$\leq 25$ & $464(44.6 \%)$ & $280(53.8 \%)$ & \\
\hline$>25$ & $576(55.4 \%)$ & $240(46.2 \%)$ & \\
\hline
\end{tabular}

** $\mathrm{p}$ value $<0.001 ;{ }^{*} \mathrm{p}$ value $<0.05$

\section{Materials and Methods}

A hospital-based cohort of 4,480 diagnosed endometrial cancer patients was identified from the database of Taiwan Cancer Registry from 2009 to 2016. The risk of endometrial cancer in Age/Grade-Deferential/Clinical or pathological Stages/Therapies was 
compared with analysis between obesity and non-obesity groups. By using these different decision tree models, prediction factors combinations for conditions of interest were identified. Moreover, a comprehensive clinical prevention approach is associated to all factors.

This study aimed to utilize data mining methods involving Support Vector Machine (SVM), Linear Discriminant Analysis (LDA), Logistic regression(LGR), C4.5, CART, Random forest (RF), and C5.0 to predict second primary endometrial cancer in obese women by different variables (Table 3). The classification accuracy of the seven methods was evaluated using receiver operating characteristic curve analysis to estimate the area under the curve (AUC) (Table 4). Accuracy, sensitivity and specificity were considered in this study (Figure 1). Support Vector Machine (SVM) classifiers operate by separating the two classes using a linear decision boundary called the hyperplane. The hyperplane is place data location that maximizes the distance between the hyperplane and instances. [5,6] Linear Discriminant Analysis (LDA) is a well-known generic method used for dimensionality reduction and classification. [7,8] Logistic regression(LGR) is the most widely used modeling approach for binary outcomes in epidemiology and medicine. The model is a part of the family of generalized linear models that explicitly models the relationship between the explanatory variable $X$ and response variable Y. [9,10] C4.5 is also a decision tree algorithm which selects the decision tree's attributes on each node based on the concept of information entropy. It adopts a greedy approach in which the decision trees are constructed in a top-down recursive divide and conquer manner. $[11,12]$ Random forest (RF) is an ensemble learning method. It generates many classification trees by selecting subsets of the given dataset and selecting subsets of predictor variables randomly, finally aggregating the results of all models to obtain a random forest.[13] The C5.0 decision tree is a classification approach which generates the tree in top-down scheme based on the information using a recursive process.[14] CART is a decision tree system which uses a binary recursive procedure to partition the data in homogenous subsets based on Gini index.[15,16]

In this study, CART can drive an ideal prediction model in obese woman (BMI > 25) (Figure 2).

Table 3. The important variables and coding in this study.

\begin{tabular}{|c|c|c|}
\hline Variable & Name & $\begin{array}{l}\text { Definition of } \\
\text { normal test data }\end{array}$ \\
\hline $\mathbf{X 1}$ & Age at Diagnosis & $<50 / \geq 50$ \\
\hline $\mathrm{X} 2$ & Grade/differentiation & $\leq 2 />2$ \\
\hline $\mathrm{X} 3$ & Tumor size & $<2 \mathrm{~cm} / \geq 2 \mathrm{~cm}$ \\
\hline $\mathrm{X} 4$ & Clinical stage Group & $<\mathrm{II} / \geq \mathrm{II}$ \\
\hline$X 5$ & Pathologic Stage Group & $<\mathrm{II} / \geq \mathrm{II}$ \\
\hline $\mathrm{X} 6$ & Surgical Margins of The Primary Site & No/Yes \\
\hline $\mathrm{X} 7$ & Date of first surgical procedure & No/Yes \\
\hline X8 & Sequence of RT and Surgery & No/Yes \\
\hline X9 & $\begin{array}{l}\text { Sequence of Locoregional Therapy and } \\
\text { Systemic Therapy(Chemotherapy) }\end{array}$ & No/Yes \\
\hline $\mathrm{X} 10$ & BMI & $\leq 25 />25$ \\
\hline $\mathbf{Y}$ & SPC & No/Yes \\
\hline
\end{tabular}


Table 4. Classification results of the seven methods.

\begin{tabular}{ccccc}
\hline Methods & Accuracy & Sensitivity & Specificity & AUC \\
\hline SVM & 0.875 & 0.8919 & 0.8692 & 0.8767 \\
LDA & 0.7014 & 0.9459 & 0.6168 & 07835 \\
LGR & 0.7431 & 0.8649 & 0.7009 & 0.8047 \\
C4.5 & 0.8819 & 0.9065 & 0.8108 & 0.914 \\
CART & 0.8403 & 0.8108 & 0.8505 & 0.9153 \\
RF & 0.7778 & 0.9459 & 0.7196 & 0.8881 \\
C5.0 & 0.7153 & 0.9459 & 0.6355 & 0.851 \\
\hline
\end{tabular}

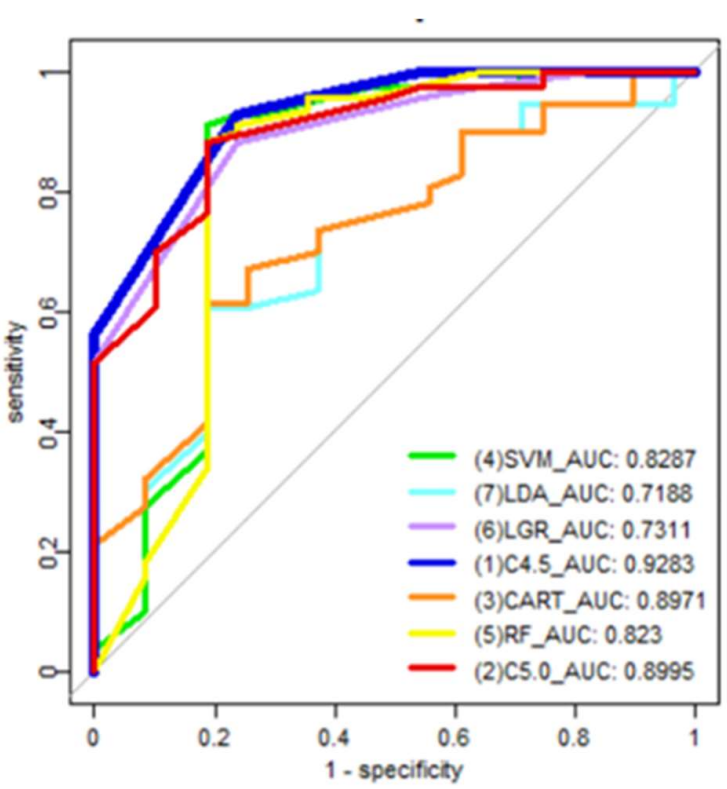

Figure 1. ROC curves of the seven methods.

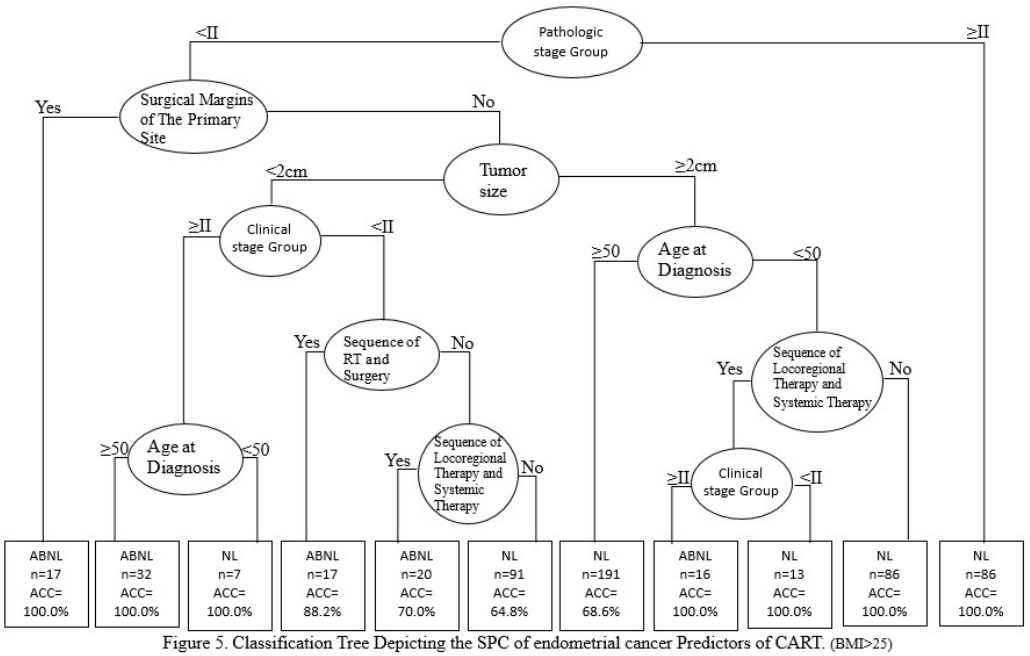

Figure 2. Classification tree depicting the SPCs of endometrial cancer predictors of CART (BMI>25) 


\section{Results}

During the study period, 530 patients were diagnosed with SPCs in endometrial cancers.

All subjects were divided into 13 subgroups, from the root node to leaf nodes, through different branches. As previously explained, clinical stage variable has a great influence on the interpretation of the SPCs and was, therefore, identified as the root node of the classified decision tree.

The first-rule decision tree was obtained from the following determining factors: pathologic stage $(<\mathrm{II})+$ surgical margins involvement (Yes), and the accuracy obtained was 1 across 17 samples. The second-rule decision tree was obtained from the following determining factors: pathologic stage $(<\mathrm{II})+$ surgical margins involvement $(\mathrm{No})+$ tumor size $(<2 \mathrm{~cm})+$ clinical stage $(\geq \mathrm{II})+$ age at diagnosis $(\geq 50)$, and the accuracy obtained was 1 across 32 samples. The fourth-rule decision tree was obtained from the following determining factors: pathologic stage $(<\mathrm{II})+$ surgical margins involvement $(\mathrm{No})+$ tumor size $(<2 \mathrm{~cm})+$ clinical stage $(<\mathrm{II})+$ sequence of radiotheraoy/Surgery (Yes), and the accuracy obtained was 0.882 across 17 samples. The fifth-rule decision tree was obtained from the following determining factors: pathologic stage $(<\mathrm{II})+$ surgical margins involvement $(\mathrm{No})+$ tumor size $(<2 \mathrm{~cm})+$ clinical stage $(<\mathrm{II})+$ sequence of radiotherapy/surgery $(\mathrm{No})+$ sequence of locoregional/systemic therapy(Yes), and the accuracy obtained was 0.7 across 20 samples. The eighth-rule decision tree was obtained from the following determining factors: pathologic stage $(<\mathrm{II})+$ surgical margins involvement $(\mathrm{No})+$ tumor size $(\geq 2 \mathrm{~cm})+$ age at diagnosis $(<50)+$ sequence of locoregional/systemic therapy (Yes) + clinical stage $(\geq \mathrm{II})$, and the accuracy obtained was 1 across 16 samples.

Therefore, the decision tree can be divided into abnormal (ABNL)(SPC) or normal (NL)(Non-SPC) situations. The accuracy ranged from $68.5 \%$ to $100 \%$.(Figure 2) There're 5 rules related to prediction models of SPCs in endometrial cancer in obese woman (Table 5).

For obese woman (BMI $>25)$, age $(\geq 50$ year-old, $\mathrm{p}=0.019)$, tumor size $(\geq 2 \mathrm{~cm}, \mathrm{p}<0.001)$, clinical and pathological stage $(<\mathrm{II}, \mathrm{p}<0.001)$, surgery (Yes, $\mathrm{p}=0.014)$, and sequence of radiotherapy/surgery (No, $\mathrm{p}<0.001$ ) had higher risk of SPCs in endometrial cancer (Table 6).

Table 5. The Summarized rules of condition variables. (BMI>25). 


\section{Rules No.}

SPCs /Observed

(n)

$17 / 20$

$100.0 \%$

1 Pathologic stage (<II) + Surgical Margins involvement (Yes)

$32 / 40$

$100.0 \%$

2

Pathologic stage $(<\mathrm{II})+$ Surgical Margins involvement (No) + Tumor

size $(<2 \mathrm{~cm})+$ Clinical stage $(\geq \mathrm{II})+$ Age at Diagnosis $(\geq 50)$

4

Pathologic stage $(<\mathrm{II})+$ Surgical Margins involvement (No) + Tumor size $(<2 \mathrm{~cm})+$ Clinical stage $(<\mathrm{II})+$ Sequence of Radiotherapy (Yes)

$17 / 24$

$88.2 \%$

Pathologic stage $(<\mathrm{II})+$ Surgical Margins involvement $(\mathrm{No})+$ Tumor

5 size $(<2 \mathrm{~cm})+$ Clinical stage $(<\mathrm{II})+$ Sequence of Radiotherapy $(\mathrm{No})+$ Se-

$20 / 28$

$70.0 \%$ quence of Locoregional/Systemic Therapy(Yes)

Pathologic stage $(<\mathrm{II})+$ Surgical Margins involvement (No) + Tumor

$8 \quad \operatorname{size}(\geq 2 \mathrm{~cm})+$ Age at Diagnosis $(<50)+$ Sequence of Locoregional/Sys-

$16 / 20$

$100.0 \%$ temic Therapy $($ Yes $)+$ Clinical stage $(\geq \mathrm{II})$

Table 6. The subject demographics of independent predictors of SPCs in endometrial cancer. 


\begin{tabular}{|c|c|c|c|c|c|c|}
\hline \multirow[t]{2}{*}{ Characteristics } & \multicolumn{3}{|c|}{$\mathrm{BMI} \leq 25$} & \multicolumn{3}{|c|}{$\mathrm{BMI}>25$} \\
\hline & Without SPCs & With SPCs & $\mathrm{p}$ value & $\begin{array}{l}\text { Without } \\
\text { SPCs }\end{array}$ & With SPCs & p value \\
\hline $\mathrm{N}(\%)$ & $560(66.7 \%)$ & $280(33.3 \%)$ & & $480(66.7 \%)$ & $240(33.3 \%)$ & \\
\hline Age at Diagnosis & & & 0.117 & & & $0.019^{*}$ \\
\hline$<50$ year-old & $190(33.9 \%)$ & $200(71.4 \%)$ & & $161(33.5 \%)$ & $60(25.0 \%)$ & \\
\hline$\geq 50$ year-old & $370(66.1 \%)$ & $80(28.6 \%)$ & & $319(66.5 \%)$ & $180(75.0 \%)$ & \\
\hline Grade/Differentiation & & & 0.004 & & & 0.698 \\
\hline 1,2 & $377(67.3 \%)$ & $160(57.1 \%)$ & & $313(65.2 \%)$ & $160(66.7 \%)$ & \\
\hline others & $18332.7 \%)$ & $120(42.9 \%)$ & & $167(34.8 \%)$ & $80(33.3 \%)$ & \\
\hline Tumor Size & & & 0.144 & & & $<0.001^{* *}$ \\
\hline$<2 \mathrm{~cm}$ & $172(30.7 \%)$ & $100(35.7 \%)$ & & $102(21.3 \%)$ & $120(50.0 \%)$ & \\
\hline$\geq 2 \mathrm{~cm}$ & $388(69.3 \%)$ & $180(64.3 \%)$ & & $378(78.7 \%)$ & $120(50.0 \%)$ & \\
\hline Clinical Stage & & & $<0.001$ & & & $<0.001^{* *}$ \\
\hline$<\mathrm{II}$ & $463(82.7 \%)$ & $120(42.9 \%)$ & & $385(80.2 \%)$ & $160(66.7 \%)$ & \\
\hline$\geq \mathrm{II}$ & $97(17.3 \%)$ & $160(57.1 \%)$ & & $95(19.8 \%)$ & $80(33.3 \%)$ & \\
\hline Pathologic Stage & & & 0.032 & & & $<0.001^{* *}$ \\
\hline$<\mathrm{II}$ & $446(79.6 \%)$ & $240(85.7 \%)$ & & $384(80.0 \%)$ & $240(100.0 \%)$ & \\
\hline$\geq \mathrm{II}$ & $114(20.4 \%)$ & $40(14.3 \%)$ & & $96(20.0 \%)$ & $0(0.0 \%)$ & \\
\hline Surgical Margins involved & & & 0.648 & & & 0.170 \\
\hline No & $515(92.0 \%)$ & $260(92.9 \%)$ & & $424(88.3 \%)$ & $220(91.7 \%)$ & \\
\hline Yes & $45(8.0 \%)$ & $20(7.1 \%)$ & & $56(11.7 \%)$ & $20(8.3 \%)$ & \\
\hline Surgical procedures & & & 0.002 & & & $0.014^{*}$ \\
\hline No & $19(3.4 \%)$ & $0(0.0 \%)$ & & $12(2.5 \%)$ & $0(0.0 \%)$ & \\
\hline Yes & $541(96.6 \%)$ & $280(100.0 \%)$ & & $468(97.5 \%)$ & $240(100.0 \%)$ & \\
\hline Radiotherapy/Surgery & & & $<0.001$ & & & $<0.001^{* *}$ \\
\hline No & $332(3.4 \%)$ & $120(42.9 \%)$ & & $249(51.9 \%)$ & $180(75.0 \%)$ & \\
\hline Yes & $228(96.6 \%)$ & $160(57.1 \%)$ & & $231(48.1 \%)$ & $60(25.0 \%)$ & \\
\hline $\begin{array}{l}\text { Locoregional/Systemic } \\
\text { Therapy }\end{array}$ & & & $<0.001$ & & & 0.867 \\
\hline No & $402(71.8 \%)$ & $160(57.1 \%)$ & & $317(66.0 \%)$ & $160(66.7 \%)$ & \\
\hline Yes & $158(28.2 \%)$ & $120(42.9 \%)$ & & $163(34.0 \%)$ & $80(33.3 \%)$ & \\
\hline
\end{tabular}

${ }^{* *} \mathrm{p}$ value $<0.001 ;{ }^{*} \mathrm{p}$ value $<0.05$

\section{Discussion}


Recent advancements in diagnostic and therapeutic methods have increased the overall survival of patients with cancers. As cancer survival rates increased, the incidence of second primary cancers has gradually increased at the same time. However, this phenomenon is due to multiple factors such as genetic or environmental factors, and developments of new anti-cancer drugs. In the present study, SPCs in endometrial cancers was observed in $11.6 \%$ of 4480 patients who had been ever diagnosed as primary endometrial cancer. Obesity (BMI $\geq 30 \mathrm{~kg} / \mathrm{m} 2)$ is the strongest risk factor for primary EC. For every $5 \mathrm{~kg} / \mathrm{m} 2$ increase in BMI, there is a $60 \%$ increased risk of EC, with a BMI above $25 \mathrm{~kg} / \mathrm{m} 2$ doubling the risk and a BMI above $30 \mathrm{~kg} / \mathrm{m} 2$ tripling the risk.[17] However, obesity could not be a crucial risk factor in second primary endometrial cancers.[18]

Currently, there is no resolution in early screening for endometrial cancer; screening is unable to decreasing mortality from endometrial cancers. It will mainly detect women with low-risk tumors.[19] In literature reviews, increasing age and long-term exposure to unopposed estrogens are strong risk factors for endometrial cancer. Metabolic syndrome (obesity, diabetes) was also a well-known risk factor. It alters concentrations of insulin-like growth factor and its binding proteins.[20] Estrogen-receptor transcriptional activity can be induced by signaling by insulin-like growth factor 1 even in the absence of estradiol, which increases the incidence of endometrial cancer.[21-24] In our study, obesity seems to be an independent risk factor to prognostic factors of primary endometrial cancer. It also plays a key role in the incidence of second primary endometrial cancer.[25]

The use of preoperative radiotherapy has been abandoned because it interferes with surgical staging and there is no benefit over postoperative radiotherapy.[1] The aim of adjuvant radiotherapy is for the pelvic lymph-node regions that might contain microscopic metastasis, as well as the central pelvic region and the upper vagina. There is a consensus that patients with lesions of surgical stage IA or IB and grade 1 or grade 2 (low risk) can be treated without postoperative radiotherapy.[26] Isolated pelvic and vaginal recurrences of low-risk endometrial cancers can successfully be treated at the time of recurrence without radiotherapy. Therefore, radiotherapy usually used in advanced endometrial cancer. In our study, postoperative radiotherapy is an increasing risk factor in non-obesity group but a decreasing risk factor in obesity group.

Endometrial cancer is a surgically staged disease. The most important therapy for endometrial cancer is surgery. The surgical staging will provide the prognostic information for the survivors. In our study, most patients $(99.31 \%)$ had received surgical interventions for their endometrial cancer. All second primary endometrial cancer came from these patients. In our study, for the obesity group, early endometrial cancer (stage $<$ II) case who had received surgery without radiotherapy and systemic therapy has a higher risk for second primary endometrial cancer at her elder age $(>=50)$.

In the past, we used data mining classification techniques for building predictive model for early chronic kidney disease successfully.[27] In this study, we applied 13 prognostic factors to determine SPCs risk factors in obese women using data mining algorithms successfully.

\section{Conclusions}

Age (>50), BMI (>25), grade/differentiation, cancer stage, grade, and adjuvant therapies were used as the prognostic factors of endometrial cancer. In our study, we found these factors could be used to be predicting factors to secondary primary endometrial cancer. Obesity is an independent risk factor for secondary primary endometrial cancer. 
For obese women, there's a higher risk for endometrial cancer. In this study, the decision tree can be divided into abnormal (SPC) or normal (Non-SPC) situations in obese women of primary endometrial cancer, with the accuracy ranged from $68.5 \%$ to $100 \%$. In obese women, we also identified that age at diagnosis, tumor size, clinical and pathological stages, surgery, and the sequence of radiotherapy had important impacts on the predictivity of the models, while other predictors, such as grad/differentiation, surgical margin involvement and locoreginal/systemic therapy, were less important.

\begin{abstract}
Author Contributions: For research articles with several authors, a short paragraph specifying their individual contributions must be provided. The following statements should be used "Conceptualization, X.X. and Y.Y.; methodology, X.X.; software, X.X.; validation, X.X., Y.Y. and Z.Z.; formal analysis, X.X.; investigation, $X . X . ;$ resources, $X . X . ;$ data curation, $X . X . ;$ writing-original draft preparation, $X . X . ;$ writing - review and editing, X.X.; visualization, $X . X$. ; supervision, $X . X . ;$ project administration, X.X.; funding acquisition, Y.Y. All authors have read and agreed to the published version of the manuscript." Please turn to the CRediT taxonomy for the term explanation. Authorship must be limited to those who have contributed substantially to the work reported.
\end{abstract}

Funding: Please add: "This research received no external funding" or "This research was funded by NAME OF FUNDER, grant number XXX" and "The APC was funded by XXX". Check carefully that the details given are accurate and use the standard spelling of funding agency names at https://search.crossref.org/funding. Any errors may affect your future funding.

Acknowledgments: In this section, you can acknowledge any support given which is not covered by the author contribution or funding sections. This may include administrative and technical support, or donations in kind (e.g., materials used for experiments).

Conflicts of Interest: Declare conflicts of interest or state "The authors declare no conflict of interest." Authors must identify and declare any personal circumstances or interest that may be perceived as inappropriately influencing the representation or interpretation of reported research results. Any role of the funders in the design of the study; in the collection, analyses or interpretation of data; in the writing of the manuscript, or in the decision to publish the results must be declared in this section. If there is no role, please state "The funders had no role in the design of the study; in the collection, analyses, or interpretation of data; in the writing of the manuscript, or in the decision to publish the results".

\title{
References
}

1. Njoku K, Abiola J, Russell J, Crosbie EJ. Endometrial cancer prevention in high-risk women. Best Pract Res Clin Obstet Gynaecol. 2020 May;65:66-78. doi: 10.1016/j.bpobgyn.2019.12.005. Epub 2019 Dec 20. PMID: 32107136.

2. Amant F, Moerman P, Neven P, Timmerman D, Van Limbergen E, Vergote I. Endometrial cancer. Lancet. 2005 Aug 612;366(9484):491-505. doi: 10.1016/S0140-6736(05)67063-8. PMID: 16084259.

3. Crosbie EJ, Zwahlen M, Kitchener HC, Egger M, Renehan AG. Body mass index, hormone replacement therapy and endometrial cancer risk: a meta-analysis. Canc Epidemiol Prev Biomarkers. 2010 ;19(12):3119e30.

4. Renehan AG, Tyson M, Egger M, Heller RF, Zwahlen M. Body-mass index and incidence of cancer: a systematic review and meta-analysis of prospective observational studies. Lancet. 2008;371(9612):569e78.

5. Cortes C, VapnikV.Support-vectornetworks. Mach Learn.1995; 20(3): 273-97. https://doi.org/10.1007/BF00994018.

6. Sidey-Gibbons JAM, Sidey-Gibbons CJ. Machine learning in medicine: a practical introduction. BMC Med Res Methodol. 2019 Mar 19; 19(1):64. doi: 10.1186/s12874-019-0681-4. PMID: 30890124; PMCID: PMC6425557.

7. Johnson, R. A.; Wichern, D. W. Applied Multivariate Statistical Analysis, $4^{\text {th }}$ ed; Prentice Hall: Upper Saddle River, NJ, 1998.

8. Pham, T.-H.; Vicnesh, J.; Wei, J.K.E.; Oh, S.L.; Arunkumar, N.; Abdulhay, E.W.; Ciaccio, E.J.; Acharya, U.R. Autism Spectrum Disorder Diagnostic System Using HOS Bispectrum with EEG Signals. Int. J. Environ. Res. Public Health 2020, 17, 971.

9. Kleinbaum DG, Klein M. Introduction to logistic regression. In: Kleinbaum DG, Klein M, editors. Logistic regression: a selflearning text. New York: Springer; 2010. pp. 1-39.

10. Levy JJ, O'Malley AJ. Don't dismiss logistic regression: the case for sensible extraction of interactions in the era of machine learning. BMC Med Res Methodol. 2020 Jun 29;20(1):171. doi: 10.1186/s12874-020-01046-3. PMID: 32600277; PMCID: PMC7325087.

11. Quinlan, J. R. C. Programs for Machine Learning; Vol. 4.5; Morgan Kaufmann Publishers, 1993. 
12. Dima, S.; Wang, K.-J.; Chen, K.-H.; Huang, Y.-K.; Chang, W.-J.; Lee, S.-Y.; Teng, N.-C. Decision Tree Approach to the Impact of Parents' Oral Health on Dental Caries Experience in Children: A Cross-Sectional Study. Int. J. Environ. Res. Public Health 2018, 15, 692.

13. Esmaily H, Tayefi M, Doosti H, Ghayour-Mobarhan M, Nezami H, Amirabadizadeh A. A Comparison between Decision Tree and Random Forest in Determining the Risk Factors Associated with Type 2 Diabetes. J Res Health Sci. 2018 Apr 24;18(2):e00412. PMID: 29784893.

14. Peng J, Chen C, Zhou M, Xie X, Zhou Y, Luo CH. A Machine-learning Approach to Forecast Aggravation Risk in Patients with Acute Exacerbation of Chronic Obstructive Pulmonary Disease with Clinical Indicators. Sci Rep. 2020 Feb 20;10(1):3118. doi: 10.1038/s41598-020-60042-1. Erratum in: Sci Rep. 2021 Mar 2;11(1):5324. PMID: 32080330; PMCID: PMC7033165.

15. Breiman, L.; Friedman, J. H.; Olshen, R. A.; Stone, C. J. Classification and Regression Trees; CA: Wadsworth, 1984.

16. Sun, Z.; Wang, J.; Chen, Y.; Lu, H. Influence Factors on Injury Severity of Traffic Accidents and Differences in Urban Functional Zones: The Empirical Analysis of Beijing. Int. J. Environ. Res. Public Health 2018, $15,2722$.

17. Calle EE, Rodriguez C, Walker-Thurmond K, Thun MJ. Overweight, obesity, and mortality from cancer in a prospectively studied cohort of U.S. adults. N Engl J Med 2003; 348: 1625-38.

18. Haraga J, Nakamura K, Haruma T, Nyuya A, Nagasaka T, Masuyama H. Molecular Characterization of Second Primary Endometrial Cancer. Anticancer Res. 2020 Jul;40(7):3811-3818. doi: 10.21873/anticanres.14370. PMID: 32620620.

19. Gerber B, Krause A, Muller H, et al. Ultrasonographic detection of asymptomatic endometrial cancer in postmenopausal patients offers no prognostic advantage over symptomatic disease discovered by uterine bleeding. Eur J Cancer 2001; 37: 64-71

20. Potischman N, Hoover RN, Brinton LA et al. Case-control study of endogenous steroid hormones and endometrial cancer. J Natl Cancer Inst 1996; 88: 1127-35.

21. Suvanto-Luukkonen E, Sundstrom H, Penttinen J, Kauppila A, Rutanen EM. Insulin-like growth factor-binding protein-1: a biochemical marker of endometrial response to progestin during hormone replacement therapy. Maturitas 1995; 22: 25562.

22. Ayabe T, Tsutsumi O, Sakai H, et al. Increased circulating levels of insulin-like growth factor-I and decreased circulating levels of insulin-like growth factor binding protein-1 in postmenopausal women with endometrial cancer. Endocr J 1997; $44 ; 419-24$.

23. Adesanya OO, Zhou J, Samathanam C, Powell-Braxton L, Bondy CA. Insulin-like growth factor 1 is required for G2 progression in the estradiol-induced mitotic cycle. Proc Natl Acad Sci USA 1999; 96: 3287-91.

24. Lacey JV Jr, Potischman N, Madigan MP, et al. Insulin-like growth factors, insulin-like growth factor-binding proteins, and endometrial cancer in postmenopausal women: results from a US case-control study. Cancer Epidemiol Biomarkers Prev 2004; 13: 607-12.

25. Ogawa C, Nakamura K, Matsuoka H, Matsubara Y, Haraga J, Masuyama H. Risk of Gynecologic Cancer as Second versus First Primary Cancer in Japan. Acta Med Okayama. 2020 Apr;74(2):109-114. doi: 10.18926/AMO/58268. PMID: 32341584.

26. Mariani A, Webb M, Keeney G, et al. Low-risk corpus cancer: is lymphadenectomy or radiotherapy necessary? Am J Obstet Gynecol 2000; 182: 1506-19

27. Shih CC, Lu CJ, Chen GD, Chang CC. Risk Prediction for Early Chronic Kidney Disease: Results from an Adult Health Examination Program of 19,270 Individuals. Int J Environ Res Public Health. 2020 Jul 10;17(14):4973. doi: 10.3390/ijerph17144973. PMID: 32664271; PMCID: PMC7399976. 